\title{
Gene Xpert MTB/RIF Assay: A New Hope for Extrapulmonary Tuberculosis
}

\author{
Ankush Raj, Netrapal Singh, Promod K. Mehta* \\ Centre for Biotechnology, Maharshi Dayanand University, Rohtak-124001 \\ *Corresponding author-Promod K Mehta (Associate professor)
}

\begin{abstract}
The diagnostic efforts for extrapulmonary tuberculosis (TB) have been severely hampered by the lack of diagnostic tests that are accurate, simple to use and can be applied at the point of clinical care. This has been further enhanced by the widespread inability to test for drug resistance. Gene Xpert ${ }^{\circledR}$ MTB/RIF test is a novel test for the diagnosis of extrapulmonary TB and rifampicin resistance. This unique test can be used almost everywhere with minimal technical expertise, enabling diagnosis of extrapulmonary TB and simultaneous assessment of rifampicin resistance to be completed within $2 \mathrm{~h}$. In low-income countries, however, its cost, environmental limitations and difficulties involved in supply and maintenance are major obstacles. While it may be suitable for major reference hospitals, operational research is needed to evaluate the test and its additional yield above high-quality smear microscopy. In high TB burden countries like India, WHO in 2010 endorsed some guidelines for implantation of Gene Xpertß MTB/RIF test for diagnosis of TB and drug resistance.
\end{abstract}

Keywords- extrapulmonary tuberculosis, Gene Xpert® MTB/RIF, rifampicin, drug resistance, WHO.

\section{Introduction}

India has the world's largest burden of tuberculosis (TB), accounting for one-forth (24\%) of the global TB incidence. The global annual incidence estimate is 8.8 million cases, of which 1.5 million cases are from India [1]. According to Revised National Tuberculosis Control Programme, 0.8 million new cases of extrapulmonary TB (EPTB) were observed in 2010 [1]. Less than 5\% of new and previously treated TB patients were tested for MDR-TB in most countries in 2010 [1]. In India and China, almost 50\% of multidrug-resistant TB (MDR-TB) cases worldwide are estimated to occur [2]. In India, 15 to $20 \%$ of TB cases are estimated to be cases of EPTB, which affects mainly the lymph nodes, meninges, kidney, spine, and growing ends of the bones [3]. Since the causative agent Mycobacterium tuberculosis spreads from person to person, an efficient as well as rapid diagnosis is a key objective of worldwide tuberculosis control programmes.

\section{Present scenario of TB diagnosis}

The major challenge in the diagnosis of EPTB is the frequently atypical clinical presentation simulating other inflammatory and neoplastic conditions, which frequently results in a delay or deprivation of treatment. Therefore, a high degree of suspicion is required for an early diagnosis and mostly, more than one technique is necessary for the confirmation of the diagnosis. In lower-income countries, the lack of diagnostic infrastructure substantially aggravates the problem [4]. The diagnosis of EPTB cases is challenging due to inadequate clinical sample volumes available and paucibacillary nature of the biological samples. [5]. Several potential techniques have been employed for the diagnosis of EPTB specimens i.e. smear microscopic examination, culture test (both manual and automated), serological assays (both antigen and antibody detection), histological /cytological examination, Mantoux test, and PCR assays (conventional as well as Real-time PCR) [3].

The conventional Mycobacterium tuberculosis detection techniques based on microscopic examination of acid fast stained specimens (by Ziehl-Neelsen method) are still highly used for diagnostic purpose especially in TB endemic countries, although they fail to provide the required sensitivity and specificity and are unable to differentiate between $M$. tuberculosis and non-tuberculous mycobacteria (NTM) [3]. Histological examination has limitations as it can not differentiate between TB and non-TB infections caused by other related diseases e.g. sarcoidosis or NTM infections except for the demonstration of stained tubercle bacilli [6]. Culture test has variable sensitivities of 3-80\% in various clinical EPTB samples and it takes 4-8 weeks to get those results and also requires trained technicians [7]. The Mantoux test is widely employed world-wide but the false-positive results are observed due to the previous bacillus Calmette-Guérin (BCG) immunization or exposure to atypical mycobacteria and false-negative results also occur in aged individuals or immuno-suppressed individuals [7].

The reports on diagnostic tests such as enzyme-linked immunosorbent assay (ELISA), slide agglutination and PCR are available for EPTB; however, the specificities and sensitivities of these tests are variable [4]. Also, these tests require a number of manual steps, and some have a relatively long turnaround time. A series of meta-analyses has shown that nucleic acid amplification tests (NAAT) have high specificity and positive predictive value with highly variable sensitivity in cases of EPTB [8-10]. 
Although the development of real-time PCR assays has improved the speeds, sensitivities, and specificities of these molecular techniques due to its short turnaround time and automation of the procedure, the new real-time methods have still not been widely adopted [11-12]. Cobas TaqMan MTB (Roche Molecular Systems, Branchburg, NJ) is a real-time PCRbased kit using TaqMan hydrolysis probes and primers that bind to a specific, highly conserved region of the Mycobacterium genome containing the gene for $16 \mathrm{~S}$ rRNA [13].

\section{Gene Xpert MTB/RIF Assay}

Cephaid Gene Xpert MTB/RIF (Xpert) assay, which can detect M. tuberculosis complex and associated rifampin (RIF) resistance directly from clinical samples using ultrasensitive hemi-nested PCR and molecular beacon technology that operate in temperatures of $15-30^{\circ} \mathrm{C}$, even in high-humidity environments [14]. This may represent the biggest advance in TB diagnostics in past decades, as it allows the simultaneous detection of $M$. tuberculosis and RIF resistance in one assay, requires minimum handling and training, and yields results within $2 \mathrm{~h}$ [14]. These characteristics also make it a potentially attractive tool for extrapulmonary specimens.

\section{Components of Xpert assay}

The assay utilizes single-use plastic cartridges with multiple chambers that are preloaded with liquid buffers and lyophilized reagent beads necessary for sample processing, DNA extraction and heminested real time PCR [15]. The different steps of Xpert assay are following.

\subsection{Cartridge}

The assay utilizes single-use plastic cartridges with multiple chambers that are preloaded with liquid buffers and lyophilized reagent beads necessary for sample processing, DNA extraction and heminested rt-PCR [15]. The cartridge incorporates a syringe drive, a rotary drive and a filter upon which $M$. tuberculosis bacilli are deposited after being liberated from the clinical material [16]. The test platform employs a sonic horn that inserts into the cartridge base to cause ultrasonic lysis of the bacilli and release of the genetic material [16].

\subsection{Hemi-nested real time PCR}

The assay then amplifies a 192 bp segment of the $r p o B$ gene using a hemi-nested rt-PCR reaction [17]. The assay also contains lyophilized Bacillus globigii spores which serve as an internal sample processing and PCR control. The B. globigii PCR assay is multiplexed with the $M$. tuberculosis assay [18].

\subsection{Detection of RIF resistance.}

RIF resistance is particularly amenable to rapid molecular detection since $>95 \%$ of all rifampicin resistant strains contain mutations localized within the $81 \mathrm{bp}$ core region of the bacterial RNA polymerase rpoB gene, which encodes the active site of the enzyme [18]. Moreover, mutations that produce in this region are highly detective for RIF resistance, whereas susceptible isolates almost always have the same wild-type nucleotide sequence [16]. In addition, the rpo $B$ core region is flanked by $M$. tuberculosis-specific DNA sequences. Thus, by using PCR technology, it is possible to detect $M$. tuberculosis and RIF resistance simultaneously, by targeting a single gene target. Thus, the $r p o B$ gene represents a much better molecular target for the simultaneous detection of TB and the key form of drug resistance because of higher sensitivity of $r p o B$ gene than kat $\mathrm{G}$ and inh $\mathrm{A}$ for isoniazid resistance [19].

\subsection{Molecular becons}

The Xpert assay utilizes molecular beacon technology to detect DNA sequences amplified in a hemi-nested real time PCR assay [14]. Five different nucleic acid hybridization probes are used in the same multiplex reaction [18]. Each probe is complementary to a different target sequence within the rpoB gene of RIF-susceptible $M$. tuberculosis and is labeled with a differently colored fluorophore [16]. Together, these overlapping probes span the entire $81 \mathrm{bp}$ core region of the $r p o B$ gene [18].

\subsection{Detection of M. tuberculosis}

M. tuberculosis is detected by the five overlapping molecular probes that collectively are complementary to the entire 81 bp $r p o B$ core region [18]. M. tuberculosis is identified when at least two of the five probes give positive signals with a cycle threshold $\left(\mathrm{C}_{\mathrm{T}}\right)$ of $\leq 38$ cycles and that differ by no more than a prespecified number of cycles [18]. The B. globigii internal control is positive when the single $B$. globigii specific probe produces a $C_{T}$ of $\leq 38$ cycles. The standard user interface gives the signal of presence or absence of $M$. tuberculosis and the presence or absence of rifampicin resistance, and a semiquantitative estimate of the concentration of bacilli as defined by the $\mathrm{C}_{\mathrm{T}}$ range (high, <16; medium, 16-22; low, 22-28; very low, >28). Assays that are negative for M. tuberculosis and for the B. globigii internal control are reported as invalid assays [16]. 
The basis for detection of RIF resistance is the difference between the first (early $\mathrm{C}_{\mathrm{T}}$ ) and the last (late $\mathrm{C}_{\mathrm{T}}$ ) M. tuberculosisspecific beacon $\left(\Delta \mathrm{C}_{\mathrm{T}}\right)$. The system was originally configured such that resistance was reported when $\Delta \mathrm{C}_{\mathrm{T}}$ was $>3.5$ cycles and sensitive if $\leq 3.5$ cycles. Since the assay terminates after 38 cycles, the assay was deemed indeterminate for RIF resistance if the first probe $C_{T}$ is $>34.5$ cycles and the last probe has a $C_{T}$ of $>38$ cycles [18]. From May 2010 the automated detection of RIF resistance was modified using a new $\Delta \mathrm{C}_{\mathrm{T}}$ cut-off in order to improve the specificity for RIF resistance detection [20].

\section{Working of Xpert assay}

Working of Xpert assay has been shown in fig.1. Firstly clinical samples were treated with sodium hydroxide and isopropanol containing sample reagent (SR) [16]. The SR is added to the sample at 2:1 ratio and incubated at room temperature for $15 \mathrm{~min}$ [14]. This step is designed to reduce the viability of $M$. tuberculosis in sample at least $10^{6}$-fold to reduce biohazard risk [21]. The treated sample is then manually transferred to the cartridge which is loaded into the GeneXpert instrument. Subsequent processing is fully automated [16]. A schematic of the assay procedure is shown in Fig 1.

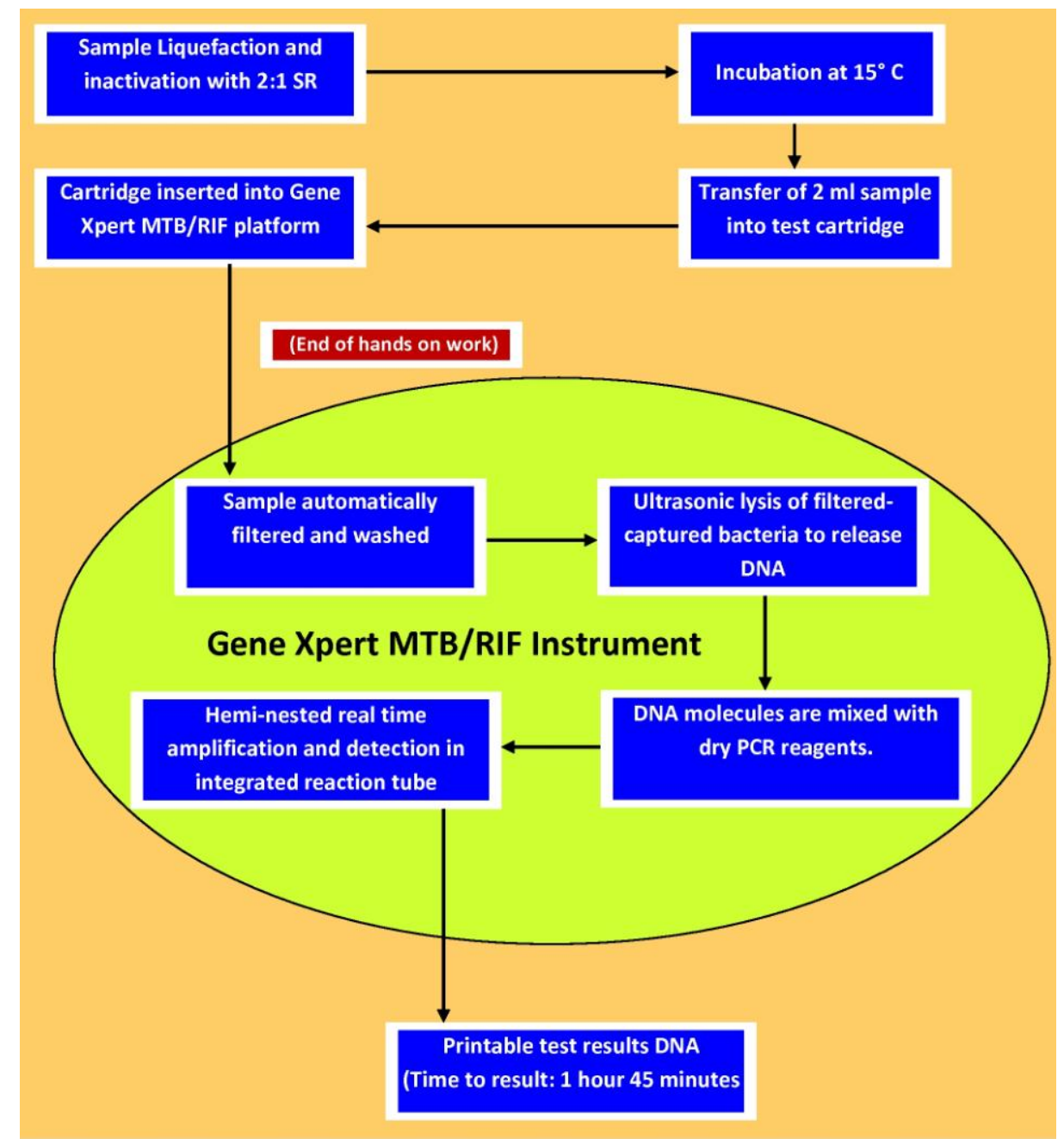

Fig.1. Various steps of Xpert Assay

\section{Sensitivity and specificity}

The specificity of the Xpert test in the diagnosis of pulmonary TB has been shown to be very high (97-100\%) in demonstration studies coordinated by the Foundation for Innovative New Diagnostics (FIND) in pulmonary samples [22]. Laboratory-based studies in Germany, France and Spain have assessed the utility of Xpert for TB diagnosis in nonrespiratory samples, including pleural fluid, gastric fluid, tissue, cerebrospinal fluid, urine and stool [16]. 521 nonrespiratory samples were detected with Xpert test having overall sensitivity of $77.3 \%$ and a specificity of $98.2 \%$, using culture as gold standard [23]. 
Preliminary analysis demonstrated excellent performance of Xpert test on fine needle aspirates samples with high sensitivity (100\%) and specificity (93.8\%) compared to MGIT culture [24]. Xpert test has the potential to significantly improve and expedite the diagnosis of smear negative and extra-pulmonary TB at both hospital and point-of-care settings in regions with overlapping HIV and TB epidemics [24].

Ligthelm et al. (2011) demonstrated the excellent diagnostic accuracy of the Xpert test in patients with tuberculous lymphadenitis with sensitivity of $96.7 \%$ and specificity of $90 \%$ [25].

Recently, Tortoli et al. (2012) investigated a large number of consecutive extra-pulmonary clinical specimens $(1,476)$ including both paediatric (494) and adult samples with overall sensitivity (81\%) and specificity (99.8\%) using .combination of culture and clinical diagnosis as gold standard [26].

Collectively, these results indicate substantial utility of Xpert for diagnosis of extra-pulmonary TB and further prospective evaluations of clinical suspects are warranted to more precisely define the utility for different forms of extrapulmonary disease.

\section{Comparative study with other assays}

From India, the Xpert was evaluated to test its utility in 547 patients with suspected extrapulmonary tuberculosis. For culture, the sensitivity was low, 53\% (150/283 specimens). Xpert sensitivity and specificity results were assessed in comparison to a composite reference standard made up of smear and culture results and clinical, radiological, and histological findings [14]. The sensitivity of the Xpert assay was 81\% (228/283 specimens) (64\% [89/138] for smear-negative cases and 96\% [139/145] for smear-positive cases), with a specificity of $99.6 \%$ [14]. Causse et al. (2011) tested a wide range of nonrespiratory samples $(\mathrm{n}=341)$ and reported a higher sensitivity of $95 \%$ and specificity of $100 \%$, outperforming the commercially available Cobas TaqMan ${ }^{\circledR}$ MTB assay [17]. In one previous study, Armand et al. (2011) demonstrated the results from just 32 nonrespiratory samples and found an overall sensitivity of 53\% that was substantially lower than that obtained using an in-house IS6110based real-time PCR assay (78\%) [27]. By contrast, the sensitivity of the Xpert assay was 75\% while the IS6110 real time assay's sensitivitiy was $0 \%$ in smear negative extrapulmonary specimens [28].

\section{RIF resistance detection}

The Xpert test has been evaluated for testing genomic DNA from M. tuberculosis isolates with 23 different commonly occurring rpoB mutations with $100 \%$ sensitivity [29]. A total of 16 of the 23 mutations caused complete failure of detection of at least one of the five probes and the remaining mutations produced a $\Delta \mathrm{CT}$ of $>3.5$ cycles. All DNA samples from RIF susceptible isolates were correctly identified as susceptible with an average $\Delta \mathrm{CT}$ of 1.8 cycles [29]. Blakemore et al. (2010) tested low concentrations of genomic DNA from 79 phylogenetically and geographically diverse strains of M. tuberculosis that included 42 rifampicin susceptible and 37 resistant strains [18]. Further experiments were done in which DNA from resistant and susceptible strains was mixed in varying ratios to assess how this impacted detection of rifampicin resistance [18]. To enable detection, between $65 \%$ and $100 \%$ of the DNA from the rifampicin-resistant isolate had to be present, depending on the mutation [18]. Whereas some mutations completely block probe hybridization, others only inhibit it. If only partial inhibition occurs, then the presence of only a small concentration of wild-type amplicon would need to be present to boost the probe signal into the normal range. Overall, this suggests that in patients with mixed infections, the Xpert assay might only detect the resistant strain if it is the predominant one present. However, selection of resistant strains during the course of standard TB treatment might lead to an apparent switch from a susceptible to a resistant phenotype when comparing baseline testing with repeat testing during treatment [14].

\section{Limitations of the Test}

The Xpert test is a major advance in TB diagnostic testing, but has few limitations such as the limited shelf-life of the diagnostic cartridges, some operating temperature and humidity restrictions, requirement for electricity supply, unknown longterm robustness, and the need for annual servicing and calibration of each machine [15]. Laboratories in low-income countries are littered with expensive equipment that no longer functions because it was inappropriate to the setting to which it was donated. Ensuring sustainable systems for long-term provision of servicing and consumables may be more important and challenging than initial implementation of the diagnostic equipment itself.

\section{Impact in Low- and Middle-Income Settings}

Despite numerous microbiological studies of improved TB diagnostic technologies, we remain remarkably ignorant of how best to implement better tests to improve patient care, of who should receive the limited capacity for better tests to maximize health impact, of how these tests may impact patient-relevant outcomes, and of how these issues vary between settings [15]. The impact of better diagnostic tests on the equity of care is largely unstudied and we don't know yet how this novel 
technology will affect the delays and costs faced by patients in their journey towards a cure for this archetypal disease of poverty.

A major issue facing low- and middle-income countries wishing to implement this technology is the high cost compared to smear microscopy. However, FIND has negotiated substantial cost reductions (relative to prices in the USA and Europe) for use in the public sector in 116 high burden low- and middle-income countries [30]. The initial capital cost for the GeneXpert instrument in these settings is considerably more than for microscopy but much lower than for conventional culture and drug susceptibility testing, especially in view of the savings from dispensing with the need for expensive biosafety equipment [31]. Instruments require annual calibration, which is likely to prove difficult in remote settings, but the cost of this may be minimized by the proposed development of a web-based system. The cost per test in early 2011 (estimated at $\$ 18$ per test) is substantially greater than for microscopy, though similar to costs for performing culture and drug susceptibility testing [16]. In the short-term, implementation of the assay would represent a substantial financial increase in diagnostic costs for national ministries of health but if the full benefits of the assay in terms of clinical outcomes and TB control are favorable, this may prove to be a wise investment.

Although, findings of Vassall et al. (2011) suggested that Xpert is a cost-effective method of TB diagnosis, compared to a base case of smear microscopy and clinical diagnosis of smear-negative TB in low- and middle-income settings where, with its ability to substantially increase case finding, it has important potential for improving TB diagnosis and control [32]. The extent of cost-effectiveness gain to TB programmes from deploying Xpert is primarily dependent on current TB diagnostic practices.

\section{WHO endorsement, costs \& implementation (WHO 2010)}

In the latter part of 2010, WHO reviewed all the available evidence regarding the Xpert assay, initially through an expert group, then through the Strategic Technical Advisory Group for TB (STAG-TB) and finally within a wider WHO consultation process. In December 2010 WHO endorsed the use of the assay [31] as follows:

- In December 2010 the WHO endorsed use of this assay as the initial diagnostic test in individuals suspected of having MDR-TB or HIVassociated TB (strong recommendation).

- WHO also endorsed use of the test as a follow-on to smear microscopy in settings where MDR-TB and HIV-associated TB were less of a concern (conditional recommendation).

- Phased implementation from 2011 onwards is recommended, leading to full scale-up thereafter. The cost of cartridges will decrease over time.

\section{Conclusion}

The Xpert technique has demonstrated a substantial capacity for the diagnosis of EPTB mostly from nonsterile fluids (gastric aspirates and urine samples), lymph nodes or abscess aspirate specimens. The high cost of this novel technology is offset to an extent by the rapid turnaround time as observed with the smear microscopic examination (less than two hours) with less biohazard problems and only minimal training required. Hence, the Xpert test not only has good sensitivity and specificity for the diagnosis of TB and detection of RIF resistance in EPTB but also perfectly fits the requirements of the Indian health care setting.

\section{References}

1. World Health Organization: Global tuberculosis control: epidemiology, strategy, financing. WHO report 2010.

2. World Health Organization. 2010. Multidrug and extensively drug-resistant TB (M/XDR-TB): 2010 global report on surveillance and response. WHO/ HTM/TB/2010.3. World Health Organization, Geneva, Switzerland.

3. S.K. Sharma and Mohan A, Extrapulmonary tuberculosis.Indian J Med Res, 120(4), 2004, 316-53.

4. S. Haldar, M. Bose, P. Chakrabarti, H.F. Daginawala, B.C. Harinath, R.S. Kashyap et al., Improved laboratory diagnosis of tuberculosis - The Indian experience. Tuberculosis, 91(5), 2011, 414-26.

5. S. Chakravorty, M.K. Sen and J.S. Tyagi, Diagnosis of extrapulmonary tuberculosis by smear, culture, and PCR using universal sample processing technology. J Clin Microbiol. 43(9), 2005, 4357-4362.

6. K. Chawla, S. Gupta, C. Mukhopadhyay, P.S. Rao and S.S. Bhat, PCR for M. tuberculosis in tissue samples. J Infect Dev Ctries. 3(2), 2009, 83-7.

7. C. Lange and T. Mori, Advances in the diagnosis of tuberculosis. Respirology, 15(2), 2010, 220-40. 
8. M. Pai, L.L. Flores, N. Pai, A. Hubbard, L.W. Riley and J.M. Jr. Colford, Diagnostic accuracy of nucleic acid amplification tests for tuberculous meningitis: a systematic review and meta-analysis. Lancet Infect. Dis. 3(10), 2003, 633-643.

9. M. Pai, L.L. Flores, N. Pai, A. Hubbard, L.W. Riley and J.M. Jr. Colford, Nucleic acid amplification tests in the diagnosis of tuberculous pleuritis: a systematic review and meta-analysis. BMC Infect Dis. 4, 2004, 6.

10. J. Dinnes, J. Deeks, H. Kunst, A. Gibson, E. Cummins, N. Waugh, F. Drobniewski and A. Lalvani, A systematic review of rapid diagnostic tests for the detection of tuberculosis infection. Health Technol Assess. 11(3), 2007, 1-196.

11. T. Takahashi, M. Tamura, Y. Asami, E. Kitamura, K. Saito, T. Suzuki, S.N. Takahashi, K. Matsumoto, S. Sawada, E. Yokoyama and T. Takasu, Novel wide-range quantitative nested real-time PCR assay for Mycobacterium tuberculosis DNA: clinical application for diagnosis of tuberculous meningitis. J Clin Microbiol. 46(5), 2008, 1698-707.

12. K. Baba, S. Pathak, L. Sviland, N. Langeland, A.A. Hoosen, B. Asjo, A.M. Dyrhol-Riise and T. Mustafa, Real-time quantitative PCR in the diagnosis of tuberculosis in formalin-fixed paraffin-embedded pleural tissue in patients from a high HIV endemic area. Diagn Mol Pathol, 17(2), 2008, 112-7.

13. J.H. Kim, Y.J. Kim, C.S. Ki, J.Y. Kim and N.Y. Lee, Evaluation of Cobas TaqMan MTB PCR for detection of Mycobacterium tuberculosis. J Clin Microbiol, 49(1), 2011, 173-6.

14. V. Vadwai, C. Boehme, P. Nabeta, A. Shetty, D. Alland and C. Rodrigues. Xpert MTB/RIF: a new pillar in diagnosis of extrapulmonary tuberculosis? J Clin Microbiol, 49(7), 2011, 2540-5.

15. C.A. Evans, GeneXpert--a game-changer for tuberculosis control? PLoS Med, 8(7), 2011.

16. S.D. Lawn and M.P. Nicol, Xpert ${ }^{\circledR}$ MTB/RIF assay: development, evaluation and implementation of a new rapid molecular diagnostic for tuberculosis and rifampicin resistance. Future Microbiol. 6(9), 2011, 1067-82.

17. M. Causse, P. Ruiz, J.B. Gutiérrez-Aroca and M. Casal, Comparison of two molecular methods for rapid diagnosis of extrapulmonary tuberculosis. J Clin Microbiol, 49(8), 2011, 3065-7.

18. R. Blakemore, E. Story, D. Helb, J. Kop, P. Banada, M.R. Owens, S. Chakravorty, M. Jones and D. Alland, Evaluation of the analytical performance of the Xpert MTB/RIF assay. J Clin Microbiol, ;48(7), 2010, 2495-501.

19. A.S. Piatek, A. Telenti, M.R. Murray, H. El-Hajj, W.R. Jr Jacobs, F.R. Kramer and D. Alland. Genotypic analysis of Mycobacterium tuberculosis in two distinct populations using molecular beacons: implications for rapid susceptibility testing. Antimicrob Agents Chemother, 44(1), 2000, 103-10.

20. C.C. Boehme, M.P. Nicol, P. Nabeta, et al. Feasibility, diagnostic accuracy, and effectiveness of decentralised use of the Xpert MTB/RIF test for diagnosis of tuberculosis and multidrug resistance: a multicentre implementation study. Lancet, 377, 2011, 1495-1505.

21. P.P. Banada, S.K. Sivasubramani, R. Blakemore, C. Boehme, M.D. Perkins, K. Fennelly and D. Alland, Containment of bioaerosol infection risk by the Xpert MTB/RIF assay and its applicability to point-of-care settings. J. Clin. Microbiol, 48, 2010, 3551-3557.

22. M.P. Nicol, L. Workman, W. Isaacs, J. Munro, F. Black, B. Eley, C.C. Boehme, W. Zemanay and H.J. Zar, Accuracy of the Xpert MTB/RIF test for the diagnosis of pulmonary tuberculosis in children admitted to hospital in Cape Town, South Africa: a descriptive study. Lancet Infect Dis, 11(11), 2011, 819-24.

23. D. Hillemann, S. Rüsch-Gerdes, C. Boehme and E. Richter, Rapid molecular detection of extrapulmonary tuberculosis by the automated GeneXpert MTB/RIF system. J Clin Microbiol, 49(4), 2011, 1202-5.

24. A. Van Rie, C. Menezes, L. Scott, K. Mellet, N. Gous, M.A. John, M. Mkhwanazi, L. Page-Shipp, W. Stevens and I. Sanne, High yield, sensitivity and specificity of Xpert MTB/RIF for M. tuberculosis detection in fine needle aspirates from HIV-infected TB suspects; Program and abstracts of the 18th Conference on Retroviruses and Opportunistic Infections; Boston, MA, USA. 2011; Abstract \#879 
25. L.J. Ligthelm, M.P. Nicol, K.G. Hoek, R. Jacobson, P.D. van Helden, B.J. Marais, R.M. Warren and C.A. Wright, Xpert MTB/RIF for rapid diagnosis of tuberculous lymphadenitis from fine-needle-aspiration biopsy specimens. $J C l i n$ Microbiol. 49(11), 2011, 3967-70.

26. E. Tortoli, C. Russo, C. Piersimoni et al., Clinical validation of Xpert MTB/RIF for the diagnosis of extrapulmonary tuberculosis. Eur Respir J, 2012 Jan 12.

27. S. Armand, P. Vanhuls, G. Delcroix, R. Courcol and N. Lemaitre, Comparison of the Xpert MTB/RIF test with an IS6110-TaqMan real-time PCR assay for direct detection of Mycobacterium tuberculosis in respiratory and nonrespiratory specimens. J. Clin. Microbiol, 49, 2011, 1772-1776

28. M.B. Miller, E.B. Popowitch, M.G. Backlund and E.P. Ager, Performance of Xpert MTB/RIF RUO Assay and IS6110 Real-Time PCR for Mycobacterium tuberculosis Detection in Clinical Samples. J Clin Microbiol. 49(10), 2011, 345862.

29. D. Helb, M. Jones, E. Story, et al., Rapid detection of mycobacterium tuberculosis and rifampin resistance by use of ondemand, near-patient technology. J. Clin. Microbiol, 48(1), 2010, 229-237.

30. WHO. Roadmap for rolling out Xpert MTB/RIF for rapid diagnosis of TB and MDR-TB. World Health Organization; Geneva: December. 2010.

31. WHO. Tuberculosis diagnostics automated DNA test. WHO endorsement and recommendations. World Health Organization; Geneva: 2010.

32. A. Vassall, S. van Kampen, H. Sohn et al., Rapid Diagnosis of Tuberculosis with the Xpert MTB/RIF Assay in High Burden Countries: A Cost-Effectiveness Analysis. PLoS Med. 8(11), 2011. 